Pensamiento Crítico N. ${ }^{\circ} 11$, pp. 33-64

\title{
Análisis comparativo de la productividad y distribución de fibra de alpaca entre Huancavelica y Puno
}

\author{
Marianella Crispin Cunya
}

\section{RESUMEN}

La fibra de alpaca es uno de los productos finales de la crianza de distintos tipos de ganado, pero la región es netamente reconocida como zona alpaquera, por lo cual la venta de fibra de alpaca es una de las fuentes permanentes de ingresos para estas familias andinas. No obstante, como es sabido, la fibra de alpaca tiene bajos precios en el mercado local debido a los bajos niveles de calidad, la presencia de intermediarios y una débil organización entre algunos de los factores limitantes.

Palabras claves: Alpaca, fibra, esquila, Huancavelica, Puno, alpaquero.

\begin{abstract}
Alpaca fiber is one of the end products of the different types of rearing livestock, but the region is clearly recognized as alpaquera zone, whereby the sale of alpaca fiber is one of the permanent sources of income for these families Andes. However as knowledge of the alpaca fiber has low prices in the local market due to low levels of quality, the presence of intermediaries and a weak organization among some of the limiting factors.
\end{abstract}

Keywords: Alpaca, fiber, shearing, Huancavelica, Puno, alpaquero. 


\section{Marianella Crispin Cunya}

\section{INTRODUCCIÓN}

El departamento de Huancavelica tiene los mayores índices de pobreza y extrema pobreza, debido a los problemas sociales que ha sufrido durante la década pasada. La principal fuente de ingresos de los pobladores en esta zona proviene de la actividad ganadera y agrícola.

La zona rural altoandina constituida como la zona alpaquera, cuya actividad principal es la producción de camélidos domésticos, abarca a los departamentos de Ancash, Apurímac, Arequipa, Ayacucho, Cusco, Huancavelica, Huánuco, Junín, La Libertad, Lima, Pasco y Puno y está conformada por aproximadamente 170,000 familias productoras y que indirectamente involucra a 1'500,000 de personas que participan de distinta manera en la cadena de valor.

\section{LA ECONOMÍA ALPAQUERA}

Los camélidos domésticos alpaca (Lama pacos) y llama (Lama glama), y sus parientes silvestres la vicuña (Vicugna vicugna) y guanaco (Lama guanicoe), son animales nativos estratégicos de la región andina, insuficientemente aprovechados desde la perspectiva de las oportunidades de mercado actuales y potencialidades hacia el futuro.

El sector socioeconómico involucrado en la crianza y manejo de camélidos sudamericanos es el más pobre y marginado de la sociedad peruana ubicada principalmente en la sierra centro-sur del área rural. La marginación que han sufrido estas poblaciones ha determinado que su calidad de vida se haya deteriorado en forma dramática, influyendo de sobremanera en los sistemas de producción pecuaria, y por la falta de una adecuada transferencia de tecnología o de una permanente asistencia técnica llega a niveles de producción de subsistencia. Es la población con los mayores porcentajes de pobreza. A su vez las regiones alpaqueras se caracterizan por la presencia de indicadores de necesidades básicas insatisfechas (NBI).

La economía rural involucra a la familia como unidad productiva, pues los padres, hijos, nietos, tíos y demás parientes laboran en la producción de alpacas, para luego

1 DESCO: Programa de apoyo a campesinos pastores de altura "Curso taller Fortalecimiento de organizaciones de base"- Huancavelica, enero de 2005. 


\section{Análisis comparativo de la productividad y distribución de fibra de alpaca entre Huancavelica y Puno}

obtener distintos derivados de ella como son la fibra, la carne, el cuero, las pezuñas y demás, las cuales posteriormente lo destinan al comercio o para uso propio. La mano de obra es totalmente informal, la mayoría de los miembros de la familia se involucran en la producción por necesidad y sus actividades no están enmarcadas bajo ningún régimen laboral. La producción de fibra de alpaca absorbe una mayor cantidad de mano de obra y en menor porcentaje el capital.

Desde el año 2003 se han venido aplicando distintas estrategias de desarrollo para las diversas zonas alpaqueras impulsadas por las instituciones públicas y privadas ubicadas en las regiones, entre las que destaca la región Puno, debido a que esta posee la mayor población de alpacas; por lo tanto, es la zona donde se concentran los programas y proyectos referidos al fortalecimiento y promoción de la producción y comercio de fibra de alpaca, sin embargo, los resultados de estos programas y proyectos no son los mismos en Puno y Huancavelica, a pesar de que Puno concentra la mayor cantidad de instituciones de intervención y sea el centro de aplicación de políticas de intervención en el sector alpaquero, aún así las familias siguen manteniéndose dentro del grupo de pobres y extremos pobres.

La comercialización rural es incipiente; en Puno ha mejorado relativamente en comparación con Huancavelica, ya que ésta carece de centros de acopio, tiene débil capacidad organizacional, además la presencia de los intermediarios abarata el precio de la fibra generando menores ingresos para el productor, pues estos intermediarios pagan por debajo del precio de mercado, mientras que en manos de la empresa industrial, el precio de la fibra es muchísimo mayor, lo que perjudica la capacidad adquisitiva en bienes básicos de consumo de la familia alpaquera.

\section{PROBLEMÁTICA DE LA ECONOMÍA ALPAQUERA}

Los camélidos sudamericanos son el sustento de los pobladores de los Andes, son fuente de alimento (carne), de vestido (fibra, piel), abono para la tierra (fecas), combustible (fecas secas) y animal de carga. El uso del animal como carne se limita al autoconsumo debido a la dureza de la carne por ser un animal viejo (luego de haber explotado todo el potencial posible de su fibra). 


\section{Marianella Crispin Cunya}

La lana o fibra es el principal producto, se caracteriza por su buena longitud, suavidad, brillo, resistencia y característica termoestáticas, que la hace ser muy cotizada en el mercado internacional.

La falta de información y capacitación de los productores con respecto del potencial económico que representa la finura de la fibra y consecuentemente, un deterioro natural y paulatino en el grosor de la misma, genera una situación de desventaja para el productor al no poder obtener mejores precios en los mercados cada vez más exigentes que demandan dicha materia prima.

También el deterioro constante de los recursos naturales (biodiversidad y variabilidad genética, pastos, agua, suelo) afecta principalmente las zonas con mayor presencia de criadores, entre los que se incluye un número considerable de mujeres, afectando la cadena productiva, así como la creciente fragilidad ecológica en las zonas productoras ante la recurrencia de diversos fenómenos climatológicos ("El Niño", nevadas, heladas, etc.).

El producto de la venta de la fibra a precios bajos y fluctuantes significa exiguos ingresos para los criadores y origina la saca indiscriminada de los animales. No existen estadísticas oficiales actualizadas del comercio y los destinos de la producción de la fibra, por lo mismo no se sabe con precisión el número de intermediarios a nivel de las regiones alpaqueras; sin embargo, se puede mencionar el avance en la conformación de centros de acopios, en los cuales la comercialización de la fibra se da por remates y por venta directa a la industria textil, a los intermediarios que participan, adquiriendo fibra categorizada, respetando los precios bases establecidos por cada centro de acopio. Es así que los ingresos de los criadores altoandinos provienen principalmente de la comercialización de la fibra y carne de estos animales (San Martín y Bryant, 1987).

\section{Huancavelica}

Huancavelica ${ }^{2}$ comprende un territorio de $22,131.47 \mathrm{~km}^{2}$ que representa el $1.9 \%$ de la superficie del Perú en el cual el hábitat de los camélidos domésticos se ubica entre los pisos Jalca y Janca, que esta entre los 3,800 a 5,000 msnm.

2 Fuente: Plan Estratégico Regional de Camélidos Sudamericanos en la Región de Huancavelica - CONACS. 


\section{Análisis comparativo de la productividad y distribución de fibra de alpaca entre Huancavelica y Puno}

\section{Características socioeconómicas}

En el Censo del 2005 su población representa el $1.71 \%$ respecto a la población nacional y tiene una densidad de $19.39 \mathrm{Hab} / \mathrm{km}^{2}$. El $30.17 \%$ en el área urbana y el $69.83 \%$ en el área rural.

Las mujeres constituyen el 51\% de la población. La Población Económicamente Activa (PEA) es $49.7 \%$; la población dedicada a la actividad agropecuaria es del $77.50 \%$.

Existe una gran deficiencia en la capacidad de organización en la producción de la fibra de alpaca, y además de la presencia de varios intermediarios y la venta de manera desordenada, por lo que la variabilidad de los precios afecta las ganancias de los productores. El mayor demandante de la fibra es el Grupo Inca Tops, pero el precio pagado por la fibra no es suficiente para cubrir costos de producción y la mayor parte de las necesidades básicas del productor del campo. En resumen, el problema se resumiría en:

- Los bajos niveles de ingresos se debe a los bajos niveles de productividad y a los inadecuados mecanismos de comercialización.

- Los bajos niveles de productividad de los pequeños productores de fibra de alpaca está dado por el manejo pecuario inadecuado expresado en los bajos niveles de mejoramiento genético, los incipientes conocimientos en la práctica sanitaria, la precaria práctica de esquila y el uso inadecuado de pastos.

- El precario mecanismo de comercialización se debe al deficiente sistema organizacional de los productores, la presencia de intermediarios, la ausencia o escasez de centros de acopio.

\section{CARENCIA DE ESTUDIOS SOBRE EL TEMA}

La ausencia de estudios en base a trabajo de campo sería la razón de los fracasos de las intervenciones de políticas en lo económico y social, acciones sin conocimiento y experiencia de la realidad desde el Gobierno Central hasta la participación de los actores directamente involucrados, que impide definir los factores claves que determinan la situación actual de la producción y comercialización de la fibra de alpaca. 


\section{Marianella Crispin Cunya}

El marco legal sobre el tema alpaquero es muy frágil. CONACS, ente del Estado que se encargaba de velar por el cumplimiento de la norma y ejecución de los diferentes programas de desarrollo de la producción de camélidos sudamericanos, actualmente ya no existe.

\section{RELACIONES FUNCIONALES DE LOS FACTORES QUE INTERVIENEN EN LA ECONOMÍA ALPAQUERA \\ Primera relación funcional}

$$
\begin{gathered}
++ \\
\mathrm{Y}=\mathrm{f}(\mathrm{P}, \mathrm{C})
\end{gathered}
$$

Donde:

Y: Nivel de ingreso de las familias alpaqueras -Variable Endógena.

P : Productividad de la fibra de alpaca - Variable Exógena.

C : Mecanismo de comercialización de la fibra de alpaca. Variable Exógena.

a. Productividad

$$
\begin{aligned}
& P=f(c) \\
& c=\text { calidad }
\end{aligned}
$$

b. mecanismos de comercialización

$$
\begin{aligned}
& C=f(p) \\
& p=\text { niveles de precios }
\end{aligned}
$$




\section{Análisis comparativo de la productividad y distribución de fibra de alpaca entre Huancavelica y Puno}

Asumimos que existe una relación directa entre las dos variables (endógenas y exógenas), de modo que a mayor desarrollo de productividad y mejor mecanismo de comercialización, los ingresos de los productores alpaqueros serán mayores.

Cuadro N. ${ }^{\circ}$ 1. Tabla de variables, indicadores y fuentes de información de la hipótesis uno.

\begin{tabular}{|l|l|l|}
\hline \multicolumn{1}{|c|}{ Variable } & \multicolumn{1}{c|}{ Indicador } & \multicolumn{1}{c|}{ Fuente de información } \\
\hline 1. Ingresos & Nivel de ingresos (S/.) & 1ria. Encuestas \\
\hline 2. Productividad & Calidad (Lb.) & $\begin{array}{l}\text { APROAL } \\
\text { CONACS Huancavelica y Puno } \\
\text { Visita de campo y entrevistas }\end{array}$ \\
\hline 3. Mecanismos de comercialización & $\begin{array}{l}\text { Nivel de precios (por categoría } \\
\text { de fibra) }\end{array}$ & $\begin{array}{l}\text { APROAL } \\
\text { CONACS Huancavelica y Puno } \\
\text { Visita de campo y entrevistas }\end{array}$ \\
\hline
\end{tabular}

Fuente: Elaboración Propia.

En la segunda y tercera relación funcional, las variables exógenas se endogenizan, por lo cual estas serían explicadas por otras variables.

\section{Segunda relación funcional}

$$
\begin{array}{cccc}
+ & + & + & + \\
\mathrm{P}=\mathrm{f}(\mathrm{Mg}, \mathrm{Ms}, & \mathrm{Pe}, \mathrm{Up})
\end{array}
$$

Donde:

P : Productividad de la fibra de alpaca - Variable Endógena

Mg : Mejoramiento Genético - Variable Exógena

Ms : Manejo Sanitario - Variable Exógena

Pe : Práctica de esquila - Variable Exógena

Up : Uso de pastos - Variable Exógena 


\section{Marianella Crispin Cunya}

La variable endógena tiene una relación directa con las cuatro variables exógenas. Para un mejor entendimiento de los factores que explican los niveles de productividad se realizó un ejercicio para endogenizar las variables explicativas. Así, tenemos:

a) Los bajos niveles de mejoramiento genético

$\mathrm{Mg}=\mathrm{f}($ Cpar, Prac $)$

$\mathrm{Mg}=$ Mejoramiento genético

Cpar $=$ Cuidado y parición de crías

Prac $=$ Prácticas de manejo genético

b) Los incipientes conocimientos en la práctica sanitaria de alpacas.

Ms $=\mathrm{f}($ Msan, ProLb, Resp, ProLd, Sv, Aem, Emf, Ce, Ci, Pm,Uf)

Ms = Manejo sanitario

Msan $=$ Métodos Sanitarios de las Alpacas

ProLb $=$ Producto y Lugares para el Baño

Resp $=$ Responsable de Dosificación

ProLd $=$ Productos y Lugares Usados para la Dosificación

$\mathrm{Sv}=$ Servicios veterinarios

Aem = Alpacas Crías y Adultas Afectadas por enfermedad

Emf $=$ Enfermedades

Ce $=$ Prácticas del control parasitario externo en adultos y críos 
Análisis comparativo de la productividad y distribución de fibra de alpaca entre Huancavelica y Puno

$\mathrm{Ci}=$ Prácticas del control parasitario interno en adultos y críos

$\mathrm{Pm}=$ Precio de los principales medicamentos

Uf $=$ Usos y frecuencia de uso en la producción de fibra de alpaca

c) La precaria práctica de esquila.

$\mathrm{Pe}=\mathrm{f}(\mathrm{He}, \mathrm{Cr}, \mathrm{Corr})$

$\mathrm{Pe}=$ Práctica de esquila

He $=$ Herramientas utilizadas para la esquila

$\mathrm{Cr}=$ La presencia de cercos

Corr $=$ Corrales de manejo

d) El uso inadecuado de pastos.

$\mathrm{Up}=\mathrm{f}($ Pca, P., Prp, Tp, Cp)

Up $=$ Uso de pastos

Pca $=$ Pastos cultivados y ahijaderos de pastos

P. = Práctica de manejo de pastos

Prp $=$ Problemas con el uso de pastos

$\mathrm{Tp}=$ Tipo de pastos

$\mathrm{Cp}=$ Características de los pastos 


\title{
Marianella Crispin Cunya
}

Cuadro $\mathbf{N} .^{\circ}$ 2. Tabla de variables indicadores y fuente de información de la hipótesis dos.

\begin{tabular}{|c|c|c|}
\hline Variable & Indicador & Fuente de información \\
\hline 1. Productividad & Calidad (Lb.) & $\begin{array}{l}\text { APROAL } \\
\text { CONACS Huancavelica y Puno } \\
\text { Visita de campo y entrevistas }\end{array}$ \\
\hline a. Mejoramiento genético & $\begin{array}{l}\text { Cuidado y parición de crías } \\
\text { Las prácticas de manejo genético de las alpacas }\end{array}$ & $\begin{array}{l}\text { APROAL } \\
\text { CONACS Huancavelica y Puno } \\
\text { Visita de campo y entrevistas }\end{array}$ \\
\hline b. Manejo sanitario & $\begin{array}{l}\text { Métodos sanitarios de las alpacas. } \\
\text { Producto y lugares para el baño. } \\
\text { El responsable de dosificación. } \\
\text { Productos y lugares usados para la dosificación. } \\
\text { Servicios veterinarios. } \\
\text { Alpacas crías y adultas afectadas por enfermedad. } \\
\text { Enfermedades. } \\
\text { Prácticas del control parasitario externo en adultos y críos. } \\
\text { Prácticas del control parasitario interno en adultos y críos. } \\
\text { Precio de los principales medicamentos. Usos y frecuencia } \\
\text { de uso en la producción de fibra de alpaca. }\end{array}$ & $\begin{array}{l}\text { APROAL } \\
\text { CONACS Huancavelica y Puno } \\
\text { Visita de campo y entrevistas }\end{array}$ \\
\hline c. Práctica de esquila & $\begin{array}{l}\text { Herramientas utilizadas para la esquila. } \\
\text { La presencia de cercos. } \\
\text { Los corrales de manejo. }\end{array}$ & $\begin{array}{l}\text { APROAL } \\
\text { CONACS Huancavelica y Puno } \\
\text { Visita de campo y entrevistas }\end{array}$ \\
\hline d. Uso de pastos & $\begin{array}{l}\text { Pastos cultivados y ahijaderos para pastos. } \\
\text { Prácticas de manejo de pastos. } \\
\text { Problemas con el uso de pastos. } \\
\text { Tipos de pastos. } \\
\text { Características de los pastos. }\end{array}$ & $\begin{array}{l}\text { APROAL } \\
\text { CONACS HVCA y PUNO } \\
\text { Visita de campo y entrevistas }\end{array}$ \\
\hline
\end{tabular}

Fuente: Elaboración propia.

\section{Tercera relación funcional}

\author{
$+\quad+$ \\ $\mathrm{C}=\mathrm{f}(\mathrm{So}, \mathrm{Ni}, \mathrm{Ca})$
}

Donde:

C : Mecanismo de Comercialización - Variable Endógena

So : Sistema Organizacional - Variable Exógena 


\section{Análisis comparativo de la productividad y distribución de fibra de alpaca entre Huancavelica y Puno}

$\mathrm{Ni}$ : Presencia de intermediarios - Variable Exógena

Ca : Centros de Acopio - Variable Exógena

La variable endógena tiene una relación directa con las variables exógenas So y Ca, y una relación indirecta con la variable Ca. Para un mejor entendimiento de los factores que explican los mecanismos de comercialización se realiza un ejercicio de endogenizar las variables explicativas. Así, tenemos:

a) Sistema Organizacional.

So $=f(F o)$

Fo $=$ Formas de organizaciones

b) Presencia de Intermediarios.

$\mathrm{Ni}=\mathrm{f}(\mathrm{Re})$

$\mathrm{Re}=$ Producción vendida a rescatistas

c) Presencia de Centros de Acopio.

$\mathrm{Ca}=\mathrm{f}(\mathrm{Nca})$

Nca $=$ Número de centros de acopio 


\section{Marianella Crispin Cunya}

Cuadro $\mathbf{N} .^{\circ}$ 3. Tabla de variables indicadores y fuente de información de la hipótesis tres.

\begin{tabular}{|l|l|l|}
\hline \multicolumn{1}{|c|}{ Variable } & \multicolumn{1}{|c|}{ Indicador } & \multicolumn{1}{c|}{ Fuente de información } \\
\hline 1. Mecanismos de comercialización. & $\begin{array}{l}\text { Nivel de precios por categoría } \\
\text { de fibra }\end{array}$ & $\begin{array}{l}\text { APROAL } \\
\text { CONACS Huancavelica y Puno } \\
\text { Visita de campo y entrevistas. }\end{array}$ \\
\hline $\begin{array}{l}\text { a. Sistema organizacional de los } \\
\text { productores. }\end{array}$ & Formas de organización & $\begin{array}{l}\text { APROAL } \\
\text { CONACS Huancavelica y Puno } \\
\text { Visita de campo y entrevistas. }\end{array}$ \\
\hline $\begin{array}{l}\text { b. La presencia de intermediarios. } \\
\text { c. La ausencia o escasez de centros } \\
\text { de acopio. }\end{array}$ & $\begin{array}{l}\text { Porcentaje de la producción } \\
\text { Cendida a los rescatistas. }\end{array}$ & $\begin{array}{l}\text { APROAL } \\
\text { CONACS Huancavelica y Puno } \\
\text { Visita de campo y entrevistas. }\end{array}$ \\
\hline
\end{tabular}

Fuente: Elaboración propia.

\section{COMPARACIÓN DE ECONOMÍAS ALPAQUERAS}

Se realiza el análisis comparativo. De manera que los niveles de productividad y las características del sistema de comercialización de la economía alpaquera de la región de Huancavelica serán valorados con relación a los niveles alcanzados por la región de Puno. Tres han sido los criterios que permitieron elegir como referente a Puno:

- Los niveles de pobreza similares

- Economía alpaquera: Huancavelica y Puno pertenecen a la zona que alberga la población de alpacas en el Perú, ambas ubicadas en la sierra, tienen como unidad productiva a la alpaca y como productor al campesino.

- El mayor desarrollo de Puno en términos de productividad y sistema de comercialización de fibra de alpaca. 


\section{Análisis comparativo de la productividad y distribución de fibra de alpaca entre Huancavelica y Puno}

\section{ANÁLISIS DE RESULTADOS}

a. Los bajos niveles de ingreso se deben a los bajos niveles de productividad e inadecuados mecanismos de comercialización.

Para medir los niveles de productividad de la fibra de alpaca el indicador es la calidad de la fibra. La calidad se categoriza según la finura o tipo de fibra como son: extrafina, fina, semifina y gruesa. Naturalmente, a mayores niveles de productividad le corresponderá una mayor producción de fibra fina y extrafina y a menores niveles una producción de fibra gruesa, esto expresado en menor precio asignado en el mercado.

Como puede apreciarse en el Gráfico N. ${ }^{\circ}$, para Huancavelica en ambos años de análisis -2005 y 2006- persiste la obtención de fibra gruesa y semifina, y los precios no serán muy favorables; poca es la obtención de la fibra extrafina y fina, cuyos precios serían más elevados.

En Puno hay una mayor participación de la fibra fina y semifina y menos de la fibra gruesa y extrafina. En Puno persiste la obtención a gran escala de fibra de regular calidad como es la semifina, o sea, falta desarrollar mejores condiciones de productividad. (Ver Gráfico N. ${ }^{\circ}$ 2). Puno se encuentra en mejores condiciones que Huancavelica. 


\section{Marianella Crispin Cunya}

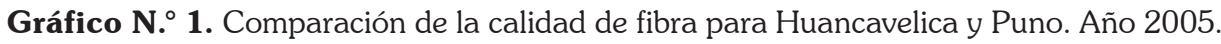

\section{TIPO DE FIBRA TOTAL REGIÓN HUANCAVELICA - 2005}

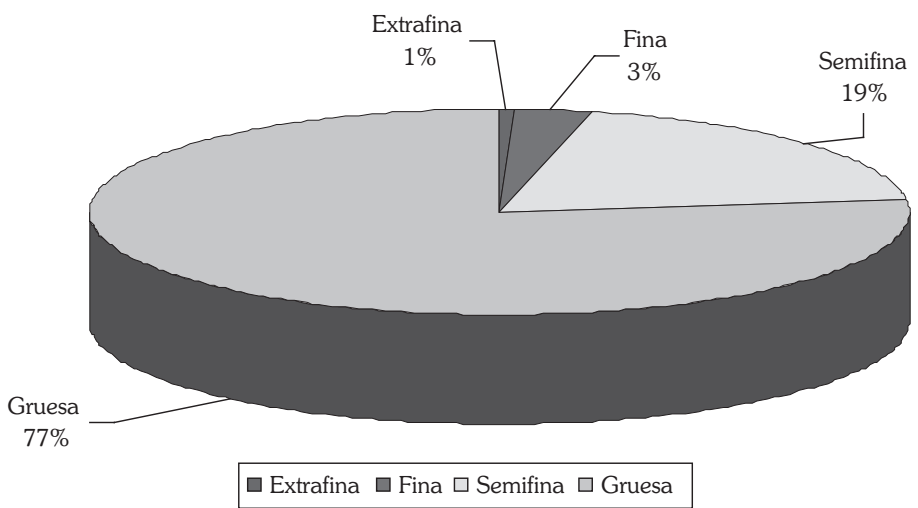

TIPO DE FIBRA TOTAL REGIÓN PUNO - 2005

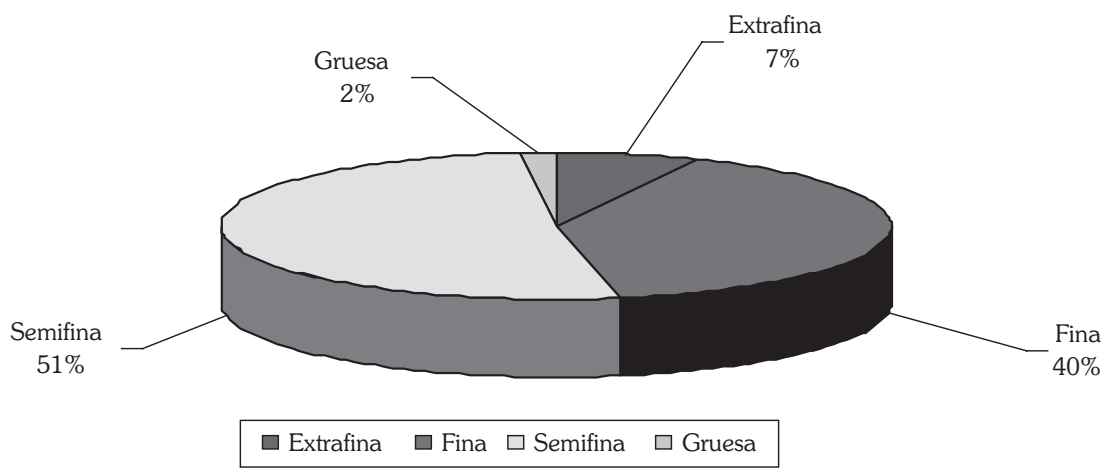

Fuente: CONACS Puno y Huancavelica

Elaboración propia. 
Análisis comparativo de la productividad y distribución de fibra de alpaca entre Huancavelica y Puno

Gráfico N. ${ }^{\circ}$ 2. Comparación de la calidad de fibra para Huancavelica y Puno. Año 2006.

TIPO DE FIBRA TOTAL REGIÓN HUANCAVELICA - 2006

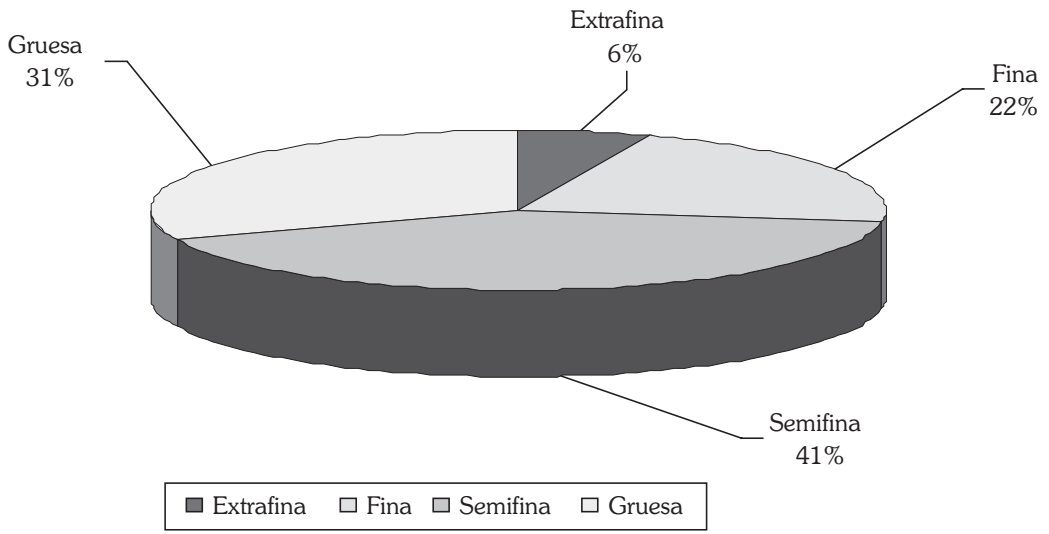

\section{TIPO DE FIBRA TOTAL REGIÓN PUNO - 2006}

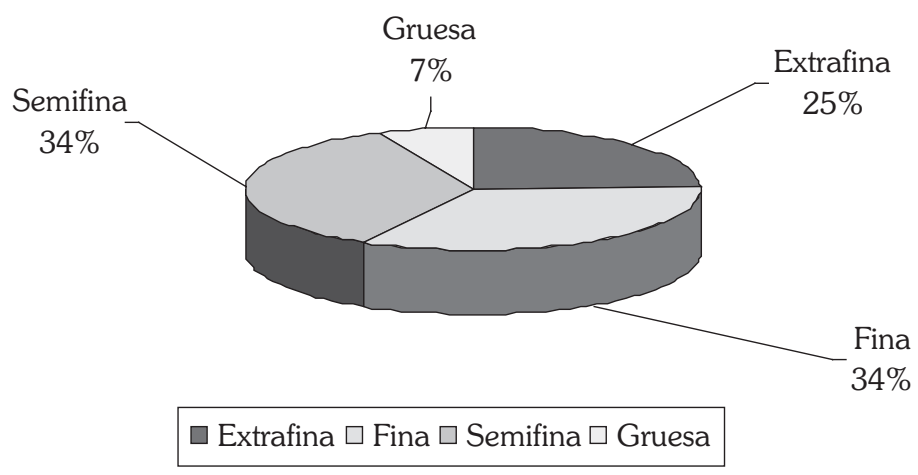

Fuente: CONACS Puno y Huancavelica

Elaboración propia. 


\section{Marianella Crispin Cunya}

La comercialización consiste en la fijación del precio de mercado para cada categoría de fibra. Los niveles de precios se ven favorecidos por la venta organizada de los pequeños productores asociados en alguna organización; acopian la fibra categorizada por tipo y color y luego pueden negociar los precios con la gran empresa proveniente de Arequipa.

Este proceso de negociación es a través de la licitación, con precios bases, y la empresa ganadora puede pagar un precio igual o mayor al precio base.

En los Cuadros N. ${ }^{\circ} 4$ y N. ${ }^{\circ} 5$ se muestra que los precios pagados por cada tipo de fibra y color en Huancavelica son menores a los precios pagados en Puno. Permite deducir la falta de intervenciones de las instituciones públicas o privadas en el fortalecimiento de las capacidades humanas y capital social para una venta organizada.

Cuadro N. ${ }^{\circ}$ 4. Comparación de los precios pagados por fibra categorizada de alpaca para Huancavelica y Puno promedio 2005 y 2006 (S/.).

\begin{tabular}{|l|c|c|c|c|c|c|c|c|c|c|c|c|}
\hline \multirow{2}{*}{ Precio / Tipo } & \multicolumn{5}{|c|}{ Huacaya } & \multicolumn{5}{c|}{ Suri } \\
\cline { 2 - 14 } & \multicolumn{2}{|c|}{ Blanco } & \multicolumn{2}{|c|}{ LF } & \multicolumn{2}{c|}{ Color } & \multicolumn{2}{c|}{ Blanco } & \multicolumn{2}{|c|}{ LF } & \multicolumn{2}{c|}{ Color } \\
\hline Región & Hvca & Puno & Hvca & Puno & Hvca & Puno & Hvca & Puno & Hvca & Puno & Hvca & Puno \\
\hline Extrafina & 8.2 & 14.0 & 7.5 & 11.5 & 4.8 & 8.5 & 6.2 & 11.0 & 5.7 & 9.5 & 5.0 & 7.5 \\
\hline Fina & 6.5 & 12.5 & 5.5 & 10.0 & 3.5 & 7.5 & 5.0 & 9.0 & 4.5 & 8.5 & 4.2 & 6.0 \\
\hline Semifina & 5.0 & 11.0 & 3.0 & 9.0 & 2.8 & 6.5 & 3.0 & 7.5 & 2.4 & 7.5 & 2.4 & 5.5 \\
\hline Gruesa & 2.0 & 7.0 & 1.5 & 6.2 & 1.5 & 3.5 & 2.0 & 6.5 & 1.8 & 5.5 & 1.5 & 3.5 \\
\hline Promedio & 5.4 & 11.1 & 4.4 & 9.2 & 3.15 & 6.5 & 4.1 & 8.5 & 3.6 & 7.8 & 3.3 & 5.6 \\
\hline
\end{tabular}

Fuente: Vecinos Perú.

CONACS Puno

Elaboración: Propia.

En conclusión, las condiciones de Puno tanto en productividad y comercio son mejores que las de Huancavelica. 
Análisis comparativo de la productividad y distribución de fibra de alpaca entre Huancavelica y Puno

Cuadro N. ${ }^{\circ}$. Cuadro de ingresos.

\begin{tabular}{|l|c|c|c|c|c|c|}
\hline \multirow{2}{*}{ Categoría } & \multicolumn{2}{|c|}{ Precio promedio } & \multicolumn{2}{c|}{ Fibra producida } & \multicolumn{2}{c|}{ Ingresos } \\
\cline { 2 - 7 } & Huancavelica & Puno & Huancavelica & Puno & Huancavelica & Puno \\
\hline Extrafina & 6,8 & 11,3 & 6 & 25 & 41,0 & 283,3 \\
\hline Fina & 5,2 & 10,0 & 22 & 33 & 113,7 & 330,0 \\
\hline Semifina & 3,6 & 8,8 & 41 & 35 & 147,6 & 309,2 \\
\hline Gruesa & 1,7 & 5,6 & 31 & 7 & 51,7 & 39,0 \\
\hline Ingreso calculado por productor al paquero & & & 353,9 & 961,5 \\
\hline
\end{tabular}

Fuente: CONACS Puno y Huancavelica.

Elaboración: Propia.

En el Cuadro N. ${ }^{\circ}$, el ingreso obtenido por productor alpaquero medio en Puno es de 2,7 veces más que el de un productor alpaquero medio de Huancavelica. En conclusión, la diferencia se explica por los desiguales niveles de productividad y por los diferentes mecanismos de comercialización de la fibra de alpaca.

b. La baja productividad de los pequeños productores de fibra de alpaca está dada por el manejo pecuario inadecuado expresado en el bajo nivel de mejoramiento genético, incipientes conocimientos en la práctica sanitaria, la precaria práctica de esquila y el uso inadecuado de pastos.

El indicador que permite explicar el manejo es la calidad de la fibra que se obtiene, la cual se expresa en el color y categoría de fibra. La calidad de la fibra como producto final del periodo de crianza de estos animales, incluye niveles de mejoramiento genético, las buenas prácticas sanitarias, el proceso de esquila y un buen uso de los pastos al momento de la crianza de las alpacas.

La esquila se inicia con la campaña de acopio de fibra para luego categorizarla por fibra y color: extrafina, fina, semifina y gruesa; para los colores son: blanco, LF (color intermedio) y color. La fibra blanca es la más demandada porque se puede adecuar a cualquier color. 


\section{Marianella Crispin Cunya}

\section{Campaña 2005}

En el Gráfico N. ${ }^{\circ} 1$ se muestra que en Huancavelica la fibra producida es de menor calidad; el $77 \%$ de la fibra producida es gruesa. Por la venta de este tipo de fibra los ingresos fueron muy bajos por el precio de este tipo de fibra. Solo el $1 \%$ de la fibra producida es extrafina, seguido de la fina con 3\% y semifina 19\%. El año 2005 el campesino no conocía sobre las ventajas del manejo de alpacas.

En Puno, la fibra producida es de regular calidad con el $51 \%$ de fibra semifina, y la fibra fina en $40 \%$. Hubo un adecuado manejo de las alpacas en las comunidades alpaqueras de Puno. Los ingresos percibidos por la venta de esta fibra les permitió cubrir sus costos de producción y obtener beneficios discretos. El 7\% de la fibra acopiada es extrafina y solo el $2 \%$ de la fibra producida es gruesa.

\section{Campaña 2006}

En Huancavelica, el 2006, como se muestra en el Gráfico N. 2, la fibra gruesa disminuyó en comparación con el año 2005, pero no se da una mejora sustancial de la calidad de la fibra, dado que la semifina es la que más participación tiene. La producción de fibra extrafina y fina sigue siendo mínima aún.

En Puno, año 2006, hay mejora sustancial en la producción de fibra de alpaca. Como se aprecia en el Gráfico N. ${ }^{\circ} 2$ en comparación con el Gráfico N.$^{\circ} 1$, la participación de la fibra por finura del total presenta un incremento en la producción de fibra de mejor calidad que es la extrafina que representa el $25 \%$ del total producido. Mientras que el $34 \%$ es la producción tanto para la semifina y fina. Solo un $7 \%$ sería la producción de fibra gruesa.

Las variables que intervienen en la obtención de la calidad de fibra categorizada son: los bajos niveles de mejoramiento genético, los incipientes conocimientos en la práctica sanitaria de alpacas, la precaria práctica de esquila, el uso inadecuado de pastos.

$$
\begin{array}{cccc}
+ & + & + & + \\
\mathrm{P}=\mathrm{f}(\mathrm{Mg}, \mathrm{Ms}, \mathrm{Pe}, \mathrm{Up})
\end{array}
$$


Análisis comparativo de la productividad y distribución de fibra de alpaca entre Huancavelica y Puno

Donde:

P : Productividad de la fibra de alpaca -Variable Endógena

Mg : Mejoramiento genético - Variable Exógena

Ms : Manejo sanitario - Variable Exógena

Pe : Práctica de esquila - Variable Exógena

Up : Uso de pastos - Variable Exógena

A continuación se detallan:

a. Mejoramiento genético

- Cuidado y parición de crías

- Prácticas de manejo genético de alpacas

- Castración de machos

- Saca

- Selección y clasificación

- Empadre

- Tratamiento sanitario

b. Manejo Sanitario

- Métodos Sanitarios de las alpacas

- Producto y lugares para el baño

- Responsable de dosificación 


\section{Marianella Crispin Cunya}

- Productos y lugares usados para la dosificación

- Servicios veterinarios

- Alpacas crías y adultas Afectadas por enfermedad

- Enfermedades

- Prácticas del control parasitario externo en adultos y críos

- Prácticas del control parasitario interno en adultos y críos

- Precio de los principales medicamentos, usos y frecuencia de uso en la producción de fibra de alpaca

c. Práctica de esquila

- Herramientas utilizadas para la esquila

- Cercos para los animales

- Corrales de manejo

- Uso de pastos

- Pastos cultivados y ahijaderos para pastos

d. Prácticas de manejo de los pastos

- Problemas con el uso de los pastos

- Tipo de pastos

- Características de los pastos en las regiones 


\section{Análisis comparativo de la productividad y distribución de fibra de alpaca entre Huancavelica y Puno}

c. El precario mecanismo de comercialización está dado por el deficiente sistema organizacional de los productores, la presencia de intermediarios, la ausencia o escasez de centros de acopio.

\section{Sistema organizacional}

La variable explicativa del sistema organizacional es el número de organizaciones existentes, en Huancavelica hay 9 organizaciones y en Puno 28 entre instituciones públicas y ONG. Se puede apreciar las condiciones bajo la cual se encuentra el capital social y humano en ambas regiones de Huancavelica y Puno.

Cuadro N. ${ }^{\circ}$ 6. Organizaciones.

\begin{tabular}{|l|l|}
\hline \multicolumn{2}{|c|}{ Organizaciones que acompañan el el proceso productivo de la fibra de alpaca } \\
\hline \multicolumn{1}{|c|}{ Huancavelica } & \multicolumn{1}{c|}{ Puno } \\
\hline Asoc. productores alpaqueros - APROAL. & 13 comités de acopio. \\
CONACS - Huancavelica, a través de su proyecto & CONACS - Puno. \\
PROALPACA. & El programa Sierra Exportadora. \\
Centro de Est. y Prom. del Desarrollo - DESCO & Dirección de Promocion Agraria del MINAG. \\
Vecinos Perú & Dirección de Crianza de camélidos. \\
Asociación Civil San Javier & El Servicio Nacional de Sanidad Agraria SENASA. \\
Centro de Innovación Tecnológica Textil - CITE & Innovación y Competitividad para el Agro Peruano \\
Textil Huancavelica. & - INCAGRO. \\
El Proyecto Piloto de Desarrollo Comunitario - & Prog. Nac. Manejo de Cuencas Hidrográficas y \\
PRODECO. & Conservación de Suelos - PRONAMACHS \\
Innovación y Competitividad para el Agro Peruano \\
- INCAGRO. & Centro de Est. y Promoción del Desarrollo - DESCO \\
& Central de Cooperación de Servicios Especiales \\
& Alpaqueras de Puno Ltda - CECOALP. \\
& Centro de Inn. Tecn. Textil - CITE Textil Puno. \\
& El Centro Peruano de Estudios Sociales CEPES \\
& Soc. Per. de Criadores de Alpacas y llamas - SPAR \\
& El Instituto Mallku. \\
CIADA. & Red de Información en Camélidos y Desarrollo de \\
& los Andes - Camelyda \\
\hline Total 9 formas de organización & \multicolumn{1}{c|}{ Total 28 formas de organización } \\
\hline
\end{tabular}

Huancavelica carece de un sistema de crédito por la falta de sujetos de crédito que garanticen la devolución de crédito, esto es por la falta de organización y un agente aval o una garantía. 


\section{Marianella Crispin Cunya}

Hay una sola forma de organización que tiene personalidad jurídica y abarca el $37,5 \%$ del total de productores alpaqueros denominada Asociación de Productores Alpaqueros - APROAL. Esta sociedad almacena las fibras acopiadas para luego venderlas a la gran empresa; el tipo de contrato entre la Asociación y la empresa es, la mayoría de veces, $50 \%$ al firmarse el contrato y el $50 \%$ luego de entrega de la cantidad en libras de fibra pactada. El monto del contrato de la campaña diciembre 2006 fue aproximadamente de S/. 180000 nuevos soles; si consideramos el tipo de contrato el monto entregado es S/. 90 000, monto que se deben repartir entre 45 comunidades aproximadamente con un promedio de 25 de familias. Es insuficiente para cubrir las necesidades básicas y el financiamiento de esta actividad productiva y para incrementar su productividad de las comunidades dentro de la asociación.

El precio que entrega el APROAL al momento de recibir la fibra de las familias o comunidades alpaqueras, es un precio referencial, el cual debe competir con los precios ofrecidos por el intermediario, quien juega con los precios. Si algún productor sabe el precio de referencia para cada tipo de fibra ofrecida por el APROAL, el intermediario ofrecerá un precio relativamente mayor a este; lo contrario sucede si el productor desconoce estos precios; esta situación es aprovechada por el intermediario para ofrecer un precio muchísimo menor al precio de referencia. Esta situación es la que comúnmente se da en aquellas comunidades fuera de la asociación y que está atomizada.

Por lo expuesto, se deduce que las condiciones de las comunidades no asociadas al APROAL o a algún tipo de asociación es aún peor; se ven obligadas a negociar con los intermediarios o rescatistas que ofrecen precios por debajo del precio de mercado asignado para cada categoría de fibra.

Otro ingreso de la familia alpaquera es con la venta de reproductores de raza; pero el desconocimiento del precio de los reproductores en el mercado obliga muchas veces al productor a vender una alpaca reproductora por debajo de su valor.

Muchos alpaqueros comprobaron el robo en peso por parte de los intermediarios, pero los precios atractivos que ofrecen éstos hace que entreguen su fibra y no se agrupen con APROAL o fomentar otro tipo de asociación. 


\section{Análisis comparativo de la productividad y distribución de fibra de alpaca entre Huancavelica y Puno}

En las ferias aprovechan los intermediarios para convencer a los productores con sus precios, mientras que algunos agentes comerciales de las empresas grandes buscan volúmenes de fibra categorizada; en este caso Huancavelica no ingresaría debido al poco volumen que presentan en las ferias y es una razón más para vender al intermediario.

Otra asociación es el Citetextil, cuya actividad principal es la de promocionar productos finales basándose en recursos propios de la zona, entre ellos los derivados de la alpaca principalmente de su fibra. Esta organización posee una balanza electrónica la cual pone a disposición del APROAL, a cambio de la venta de cierta cantidad de fibra para su proceso textil y así se registra el peso exacto en comparación con las balanzas artesanales que posee la asociación.

La empresa minera Buenaventura colabora en el proceso de acopio de la fibra en su zona de influencia, apoyando con el transporte y algunas veces financiando la capacitación de los comuneros.

El Estado, a través de su proyecto PROALPACA, viene trabajando en el fortalecimiento de las capacidades productivas a través de capacitaciones sobre el buen manejo de alpacas, las ventajas que ofrece la adecuada organización entre las comunidades, talleres en los cuales involucran a los productores o líderes de cada comunidad. INCAGRO colabora en el fortalecimiento de las capacidades de producción, gestión y comercialización sin que su intervención sea muy difundida.

Entre las ONG presentes en la región se encuentra DESCO, que busca fortalecer los agentes de la producción ganadera entre ellos el de alpaca; su intervención ha sido de gran importancia durante los años en que viene operando, aunque el problema radica en que sus proyectos son pilotos y no se difunden a nivel de la región. Vecinos Perú se orienta particularmente al fortalecimiento de las capacidades de comercio; Asociación Civil San Javier; Proyecto Piloto de Desarrollo Comunitario - PRODECO, financia algunas actividades puntuales.

En Puno, el 2006, existían 13 comités reconocidos y varias cooperativas agrarias con personalidad jurídica; posiblemente a la fecha existan más comités y cooperativas; 


\section{Marianella Crispin Cunya}

en la mayoría de las comunidades pertenecientes a los distintos comités se da la categorización de la fibra. Las cooperativas agrarias por tener la condición de persona jurídica pueden llegar a solicitar crédito a alguna institución crediticia; el problema radica en el buen manejo de estos fondos para el desarrollo y fortalecimiento de las capacidades productivas.

Cada comité representante cuenta con su centro de acopio en el distrito donde reside el comité, almacena la fibra acopiada de las distintas comunidades integradas en cada comité, para luego llevarla al centro de acopio central ubicada en la ciudad de Puno donde se realiza el proceso de comercialización con la empresa que ganó la licitación.

El Comité Regional de Acopio tenía como miembro participante al CONACS, el cual se encarga de convocar a las empresas para licitación. Los comités se reúnen mensualmente para definir las estrategias a seguir, cuando es época de campaña de acopio, las reuniones son más seguidas, es ahí donde definen el precio base de venta con la participación del comité de acopio.

La comercialización es en la ciudad de Puno; sin embargo, se opta por descentralizar esta actividad y realizarla en el distrito de Macusani, provincia de Carabaya, zona con alto potencial alpaquero. Según las entrevistas realizadas durante la campaña de acopio de la fibra, solo se logra recoger un $38,3 \%$ del total producido, el resto se reparte entre los rescatistas o intermediarios y las cooperativas ${ }^{3}$.

El resultado de esta forma organizada de comercio es que los niveles de precios para las distintos tipos de fibra tienen una tendencia creciente. Además, hay una mayor concientización de los comuneros sobre el valor monetario de los tipos de fibra y de los reproductores de mayor calidad de fibra.

En las ferias mensuales llamadas K'ATOS, las comunidades ofrecen derivados de la alpaca y los intermediarios aprovechan para captar la fibra al menudeo, y las comunidades pueden hacer trato directo con los agentes de las grandes empresas textiles.

3 Se habla de totales y no se incluye en el análisis la variable perturbadora como por ejemplo el tráfico de la fibra. 


\section{Análisis comparativo de la productividad y distribución de fibra de alpaca entre Huancavelica y Puno}

El precio base se fija luego de la reunión de los 13 comités y el comité regional de acopio y es el precio de licitación. En alguna oportunidad, con altos volúmenes de fibra acopiada, la empresa trató de bajar los precios por debajo de los preestablecidos, haciendo que se venda la fibra a un precio menor. Esto trajo un malestar entre los distintos productores, quienes decidieron no entregar el 100\% de su fibra acopiada a los comités, guardando una reserva para ofrecerlas al intermediario, quien pagaría un precio igual o por encima del precio base. La mayoría de las veces se da el caso inverso donde se logra vender el total de fibra acopiada a un precio por encima del precio base.

Otras instituciones públicas presentes esos años son el Programa Nacional de Manejo de Cuencas Hidrográficas y Conservación de Suelos (PRONAMACHS) que ha venido interviniendo en los últimos años mediante la construcción de cobertizos en algunos lugares; Dirección de Promoción Agraria del MINAG; SENASA, su participación es algo limitada; Dirección de Crianza de Camélidos; MINCETUR a través del Programa de sierra exportadora.

CITE Textil de Puno contribuye al fortalecimiento de las capacidades productoras y a la difusión de productos finales elaborados de los derivados de la alpaca. CEPES, cuyo objetivo es contribuir a la modernización y desarrollo de la sociedad agraria; el Instituto Mallku que trabaja en el sur del Perú relacionado con el ámbito rural; SPAR contribuye al desarrollo de la sociedad agraria. CIADA; Red de Información en Camélidos y Desarrollo de los Andes - Camelyda; Central de Cooperación de Servicios Especiales Alpaqueras de Puno Ltda - CECOALP es una de las cooperativas existentes que negocia con la gran empresa.

Puno, el departamento con mayor población alpaquera, se convirtió en el centro de proyectos e intervenciones por parte del Estado y otras instituciones públicas y privadas y así se difunden los precios de mercado de los reproductores, además que tiene mayores ventas de reproductores a otras regiones, lo que trajo consigo que el precio de las alpacas para reproducción se eleven. 


\section{Marianella Crispin Cunya}

a. Presencia de intermediarios

Los intermediarios desarticulan el mercado limitando los precios por la venta de la fibra, pues casi siempre los productores reciben un pago por debajo del precio de mercado. Se puede apreciar que el porcentaje de la producción vendida a los rescatistas, para el caso de Huancavelica, sería el 37,5\% y para Puno sería el $6,8 \%$.

En Huancavelica el $37,5 \%$ de la producción es absorbida por los acopiadores en APROAL, que negocia con las grandes empresas como son el Grupo INCA, Michell, Santa Isabel, Sarfati, Prosur, Fibraandina. Mientras que en Puno la situación es diferente dado que el 38,3\% de la producción es acopiada a nivel de los comités de acopio, para luego venderlos a las grandes empresas.

En Huancavelica, el 37,5\% de la producción se distribuye a nivel de los rescatistas o intermediarios que compran a un precio menor a los comuneros, para luego venderlo a un precio mayor a la gran empresa. Mientras que en Puno el 6,8\% de la producción es destinada a los rescatistas o intermediarios. Una de las razones de la venta a los intermediarios es la necesidad de liquidez en la época escolar (meses de marzo y abril), que se relaciona directamente con la venta de fibra en épocas no típicas de campaña de esquila, porque el dinero obtenido en la campaña de esquila y acopio clásico de noviembre-diciembre no es suficiente para cubrir sus necesidades durante el año. En la campaña, los precios ofrecidos por los intermediarios son mayores a los que ofrece la empresa que ganó la licitación de compra de fibra, lo que debilita la capacidad de negociación de la asociación frente a la empresa textil, y origina que este mercado de la fibra sea altamente vulnerable, y especulativo en precios.

El $15 \%$ de la fibra producida es distribuida entre los agentes comerciales de la industria arequipeña. En Huancavelica hay varias tiendas de estos agentes, como son del Grupo Inca, Michell y Sarfati y funcionan todos los días. Allí acuden los propios productores a vender su fibra (esta venta se da de manera atomizada dado que el productor vende su fibra en promedio 4 a 10 libras). Para Puno es el mismo porcentaje de $15 \%$ de la fibra distribuida a nivel de los agentes comerciales, el cual 


\section{Análisis comparativo de la productividad y distribución de fibra de alpaca entre Huancavelica y Puno}

Gráfico $\mathbf{N}{ }^{\circ}$ 3. Porcentaje de distribución de la fibra de alpaca en Huancavelica.

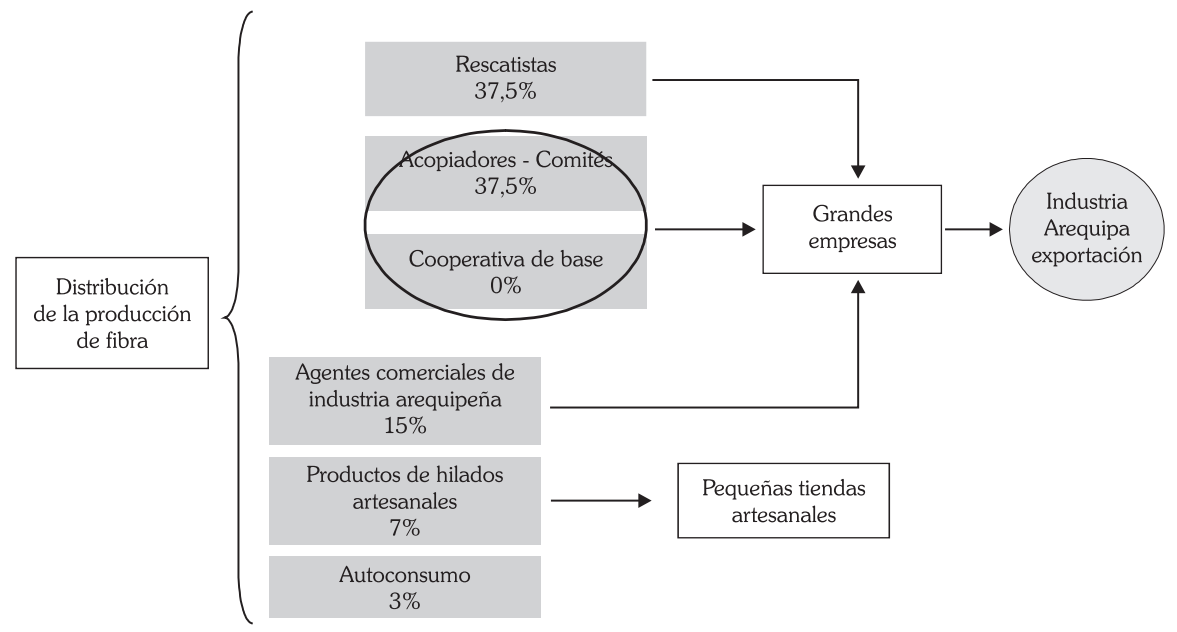

Elaboración propia.

Gráfico $\mathbf{N}^{\circ}$ 4. Porcentaje de distribución de la fibra de alpaca en Puno.

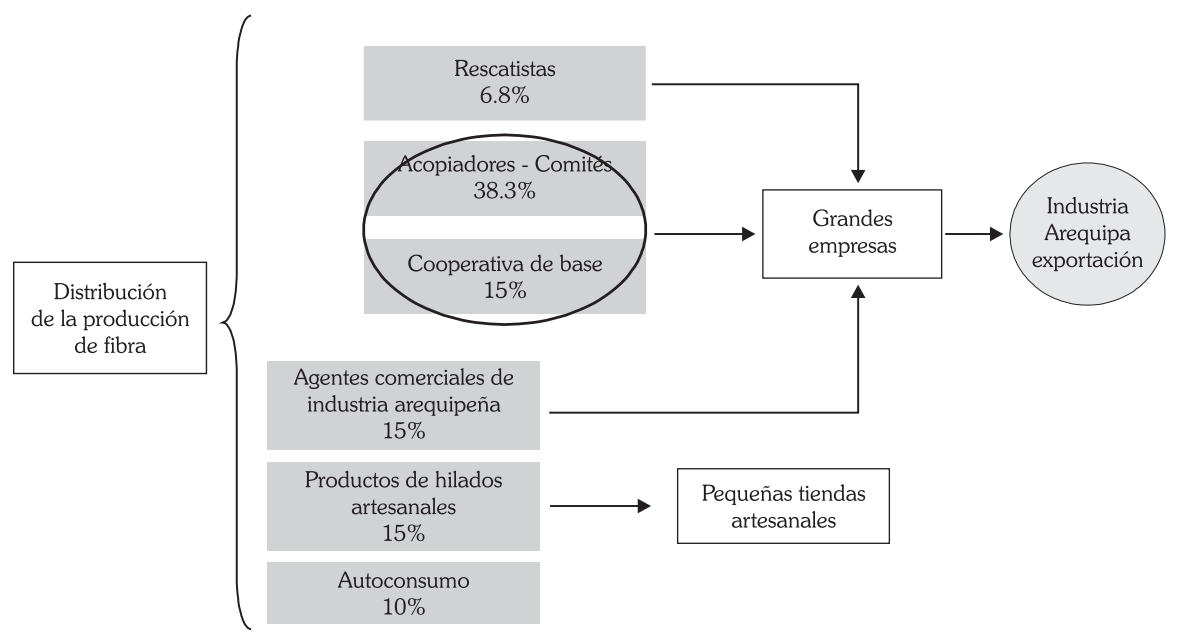

Elaboración propia. 


\section{Marianella Crispin Cunya}

tiene la misma forma que la de Huancavelica. En esta zona el 7\% de la producción de fibra se queda en manos de los artesanos, para producir textiles, carteras $u$ otro tipo de artesanía. En Puno el $15 \%$ de la fibra producida es distribuida a nivel de los artesanos.

El 3\% de la producción es destinado al autoconsumo ya sea transformándolos en chompas simples o para utilizarlos como prenda de trueque o algún uso propio para el caso de Huancavelica. En Puno el porcentaje de autoconsumo es mayor, llega al $10 \%$.

No existe cooperativas de base en Huancavelica, mientras que en Puno hay varias cooperativas de base que absorben el $15 \%$ de la fibra producida. Negocian de forma directa con la gran empresa o industria arequipeña.

b. Centros de acopio

La variable explicativa sería el número de centros de acopio, para el caso de Huancavelica sería uno que es el APROAL, mientras que para Puno existirían 13 centros de acopio.

Cuadro N. ${ }^{\circ}$ 7. Centros de acopio.

\begin{tabular}{|l|c|l|}
\hline & Huancavelica & \multicolumn{1}{c|}{ Puno } \\
\hline $\begin{array}{l}\text { Porcentaje vendido mediante } \\
\text { centros de acopio }\end{array}$ & $37,5 \%$ & $\begin{array}{l}53,3 \% \text { entre los centros de acopio de los comités y } \\
\text { los de las cooperativas de base. }\end{array}$ \\
\hline Número de centros de acopio & $\begin{array}{l}\text { Asociación de pro- } \\
\text { ductores alpaqueros } \\
\text { APROAL* }\end{array}$ & $\begin{array}{l}\text { Macusani, Capazo, Conduriri, Huanacamaya, Mazo- } \\
\text { cruz, Paratia, Pinaya, Santa Lucía, Palaca, Laraqueri, } \\
\text { Lampa, Vila Vila, San Antonio Esquilache, Centros } \\
\text { de acopios pequeños de las cooperativas de base. }\end{array}$ \\
\hline
\end{tabular}

* Su local reside en la casa del presidente de la asociación, es decir no cuentan con un local propio.

* Elaboración propia.

Fuente: Encuesta Línea Base.

Elaboración: SANJAP, Asociación Civil San Javier del Perú. 


\section{Análisis comparativo de la productividad y distribución de fibra de alpaca entre Huancavelica y Puno}

\section{CONCLUSIONES}

- Los bajos ingresos de los productores de fibra de alpaca en Huancavelica son atribuibles a la baja calidad de fibra producida y vendida; en Puno se observan ingresos más altos debido a una mejor calidad de la fibra producida y vendida. Hay mejores niveles de productividad (calidad) de Puno respecto a Huancavelica.

- En Huancavelica se carece del adecuado conocimiento sobre manejo de alpacas, lo que se traduce en bajos niveles de productividad; en Puno hay una mejora continua del manejo genético, un buen manejo sanitario, adecuado manejo de pastos, y proceso de esquila de la fibra; esto genera mejores niveles de productividad.

- El deficiente sistema de comercialización de la fibra de alpaca en Huancavelica se nota en la falta de capacidad organizativa de los productores, poca presencia de instituciones de intervención y la existencia de intermediarios, en contraste con Puno en donde existen organizaciones formadas por los productores, presencia de instituciones de intervención y pocos intermediarios, lo que ayuda a fortalecer los mecanismo de comercialización y la mejor articulación del mercado de la fibra en Puno.

\section{RECOMENDACIONES}

1. Para mejorar los niveles de ingresos de las comunidades alpaqueras de la región Huancavelica, es necesario orientar esfuerzos para impulsar y mejorar la calidad de la fibra, proceso que conducirá al desarrollo de la venta y generará mayores ingresos, mientras que en la región Puno se debe seguir fortaleciendo el mejoramiento de la calidad de la fibra. Para ambas regiones la producción de fibra es una oportunidad para mejorar sus ingresos y su capacidad adquisitiva.

2. Se requiere un trabajo coordinado y estrecho entre las comunidades alpaqueras y las instituciones que actúan en este sector para promover, fortalecer y difundir los beneficios del adecuado manejo de alpacas, para una mayor obtención de fibra de alta calidad, y un precio más alto. Por ello a las comunidades de Huancavelica se las debe capacitar permanentemente y brindar un mayor acceso a la información pertinente, para luego ser responsables de un adecuado manejo genético, manejo 


\section{Marianella Crispin Cunya}

de pastos, manejo sanitario y el proceso adecuado de la esquila; mientras que en Puno se debe seguir fortaleciendo y solidificando las adecuadas condiciones de manejo de alpaca.

3. Promover y fortalecer las capacidades organizativas en Huancavelica, concientizando a las unidades productoras sobre las ventajas del acopio organizado y las desventajas de la venta a los intermediarios que distorsiona el funcionamiento del mercado.

4. Es necesario formular un plan de desarrollo alpaquero, constituido por todas las políticas que puedan deducirse de las variables consideradas en cada una de las conclusiones presentadas anteriormente.

5. Es fundamental el rol desempeñado por las organizaciones, el Estado y las ONG, razón por la cual es necesario fortalecer las organizaciones alpaqueras para la comercialización y la transmisión de la tecnología entre los actores directos, sobre las unidades productivas para hacerlos especialistas en minimizar costos de consultaría y/o asesoramiento.

6. Realizar programas de capacitación con métodos enfocados a la realidad rural para adultos, teniendo en cuenta que los productores alpaqueros son analfabetos y algunos poseen estudios primarios inconclusos lo que les dificulta comunicarse adecuadamente y transmitir sus inquietudes.

7. Promover una adecuada capacitación a las mujeres para mejorar la clasificación de la fibra incorporando mayor valor agregado al producto.

8. Coordinar esfuerzos para rescatar los diferentes resultados de las actividades y funciones de los distintos programas que fueron impulsados por el CONACS de tal manera que se obtenga un diagnóstico a profundidad sobre el papel de una institución que parte del Estado que intervenga en la producción y comercio de fibra. 


\section{Análisis comparativo de la productividad y distribución de fibra de alpaca entre Huancavelica y Puno}

\section{BIBLIOGRAFÍA}

Cadenas productivas y redes de acción colectiva en Medellín y el Valle de Aburrá

Case, Kart E. Principios de microeconomía, 1997.

Centro Peruano de Estudios sociales - CEPES. Boletines informativos, 2005 y 2006.

Consejo Transitorio de Administración Regional de Huancavelica. Plan estratégico de desarrollo integral de Huancavelica 2000-2025. Huancavelica, 2000.

DESCO. Atlas del departamento de Huancavelica. Lima, 2000.

Espinoza Escriba, Mario. Análisis de producción de vicuñas en Lucanas, 1987.

Gonzáles, Vilma. Economía, sociedad y territorio, vol. III, núm. 10, 2001, 221-259.

Hernández Sampiere, Roberto. Metodología de la investigación. Tercera edición. México, 2003.

INEI y Ministerio de Agricultura. Censo nacional agropecuario - Resultados Definitivos Departamento de Huancavelica. Lima, 1996.

Mesa de Concertación de Huancavelica - MECODEH. Plan concertado y participativo para el desarrollo humano y sostenido del departamento de Huancavelica. Huancavelica, 1999.

Mesa de Concertación de Lucha Contra la Pobreza - Comité Ejecutivo Huancavelica. Plan estratégico concertado del departamento de Huancavelica (Versión Preliminar). Huancavelica, 2001.

Parkin, Michael. Teoría macroeconómica, 2002.

PROFINES-PRES. Elaboración de estudios de corredores de desarrollo en el departamento de Huancavelica. Tomos I y II. Lima, 1999. 


\section{Marianella Crispin Cunya}

Sánchez Carlessi, Hugo. Metodología y diseño en la investigación cientííica. Lima, 1985.

Selltiz; C; Jahoda, M; Deutsch, M. Métodos de investigación en las relaciones sociales. Cuarta edición. México, 1970.

Sociedad Peruana de Criadores de Alpacas y Llamas - SPAR. Boletines informativos. Lima, 2006 y 2005.

\section{INTERNET}

http://www.cepes.org.pe/cendoc/cultivos/camelidos/20070800/Miski_Paqu_11_ago_2007.doc

http://www.inia.gob.pe/Camelidos/resumen.htm

http://www.inia.gob.pe/boletin/boletin0021/QUINSACHATA.htm

http://www.cepes.org.pe/prueba_site.shtml?apc $=z z v x 1--\& s=a$

http://www.inia.gob.pe/

http://sisbib.unmsm.edu.pe/BVRevistas/veterinaria/v06_n1/faunap.htm

http://www.elcomercio.com.pe/EdicionImpresa/Html/2006-05-10/impNacional 0503107.html

http://www.walc2004. cepes.org.pe/prueba_site.shtml?apc $=e K 1 \mathrm{n}-\& \mathrm{x}=56531 \& \mathrm{~s}=\mathrm{R} \& \mathrm{cmd}[537]=$ i-537-2b920dfb65a4291811e04f024cbb7de2

http://www.tierramerica.net/2002/0616/noticias4.shtml

http://www.inia.gob.pe/Camelidos/resumen.htm 